\title{
Miniaturized flow cytometer with 3D hydrodynamic particle focusing and integrated optical elements applying silicon photodiodes
}

\author{
Michael Rosenauer • Wolfgang Buchegger • \\ Inez Finoulst $\cdot$ Peter Verhaert $\cdot$ Michael Vellekoop
}

Received: 26 May 2010/Accepted: 19 July 2010/Published online: 19 October 2010

(C) The Author(s) 2010. This article is published with open access at Springerlink.com

\begin{abstract}
In this study, the design, realization and measurement results of a novel optofluidic system capable of performing absorbance-based flow cytometric analysis is presented. This miniaturized laboratory platform, fabricated using SU-8 on a silicon substrate, comprises integrated polymer-based waveguides for light guiding and a biconcave cylindrical lens for incident light focusing. The optical structures are detached from the microfluidic sample channel resulting in a significant increase in optical sensitivity. This allows the application of standard solidstate laser and standard silicon-based photodiodes operated by lock-in-amplification resulting in a highly practical and effective detection system. The easy-to-fabricate singlelayer microfluidic structure enables independently adjustable 3D hydrodynamic sample focusing to an arbitrary position in the channel. To confirm the fluid dynamics and raytracing simulations and to characterize the system, different sets of microparticles and T-lymphocyte cells (Jurkat cell line) for vital staining were investigated by detecting the extinction (axial light loss) signal. The analytical classification via signal peak height/width demonstrates the
\end{abstract}

Electronic supplementary material The online version of this article (doi:10.1007/s10404-010-0707-z) contains supplementary material, which is available to authorized users.

M. Rosenauer $(\varangle) \cdot$ W. Buchegger $\cdot$ M. Vellekoop Institute of Sensor and Actuator Systems, Vienna University of Technology, Vienna, Austria

e-mail: michael.rosenauer@tuwien.ac.at

I. Finoulst $\cdot$ P. Verhaert

Analytical Biotechnology Group, Delft University

of Technology, Delft, The Netherlands

I. Finoulst $\cdot$ P. Verhaert

Netherlands Proteomics Centre, Delft, The Netherlands high sensitivity and sample discrimination capability of this compact low-cost/low-power microflow cytometer.

Keywords Hydrodynamic focusing - Polymer lens · Microfluidics · Flow cytometry

\section{Introduction}

In the past decades the application of microsystems technology has shown enormous potential for portable low-cost devices in the field of analytical biotechnology (Mogensen et al. 2004; Dittrich et al. 2006; Myers and Lee 2008). Besides the main motivation factors for sensor integration, cost and size, novel measurement, and sample handling methods were successfully introduced (Janasek et al. 2006; Ros et al. 2006; Sims and Allbritton 2007) and have been further developed to competitive diagnostic products (Blow 2007). Flow cytometry based on optical inspection techniques (Shapiro 2003) has been successfully used for many years due to its distinct advantages in terms of detection sensitivity, versatility, and excellent reliability (Huh et al. 2005; Ateya et al. 2008; Zhang et al. 2009). It offers a wide variety of detection platforms [e.g., fluorescence illumination: Tung et al. (2004), Bernini et al. (2006), Malic and Kirk (2007), Wu et al. (2008), Fu et al. (2008); extinction: Fu et al. (2004), Kummrow et al. (2009); and scattering measurements: Wang et al. (2004), Golden et al. (2009), Chen and Wang (2009)] to inspect relevant sample parameters. A suspension of particles or cells is introduced into a configurable fluidic system where they are hydrodynamically focused to significantly increase the detection sensitivity. Then the beads/cells are driven through an optical inspection region where they individually pass a focused incident light beam. The interaction 
with the excitation light (forward/side scattering and extinction) is typically measured by highly sensitive photodetectors, e.g., photomultiplier tubes or low-noise avalanche photodiodes. These features are traditionally combined in a commercial benchtop flow cytometer common to most clinical laboratories and biomedical research facilities. Although the detection sensitivity and analysis speed allow a high sample throughput, the costs of acquisition of these bulky instruments trouble many groups in this field. Therefore, researchers are developing integrated laboratories-on-chip to significantly miniaturize the devices and reduce the fabrication costs for disposable assays (McDonald et al. 2000; Whitesides 2006). By introducing new measurement features to the chip-based analytical system the interdisciplinary field of micrototal analysis system has good prospects to develop from an academic and research stadium to a serious successor of the larger size analytical laboratory equipment. The microflow cytometry development process can be separated into four parts:

- Fluidic interconnections between holder and chip,

- hydrodynamic sample focusing,

- optical detection path, and

- measurement circuitry.

The fluidic supply control in a microflow cytometer is often achieved by external pressure-driven syringe pumps. To ensure and simplify the fluidic interconnections between chip and syringes, multiple strategies, e.g., custom-made plastics holder or steel tubing platform (Blow 2007), have been proposed in the past years. Also on-chip pumping systems have been successfully integrated (pneumatic valves and peristaltic pumps (Wang and Lee 2006) and electrokinetic techniques (Xuan and Li 2005) but are used with reservations in the field of microfluidic cytometry due to additional constraints in terms of fabrication and flow behavior.

After the sample suspension is introduced and driven through the microfluidic channel system different approaches can be followed to align the particles for a reproducible optical inspection. Due to the difficulties to fabricate a round-shaped nozzle to generate a cylindrical sheath flow around the sample (applied in larger commercial cytometers) other focusing techniques are needed, such as focusing sheath fluids (Blankenstein and Darling Larsen 1998; McClain et al. 2001) and dielectrophoretic alignment ( $\mathrm{Yu}$ et al. 2005). The main objective for flow focusing, producing a stable aligned particle stream, makes it necessary to develop sheathing in more than one dimension. Different research labs successfully implemented various approaches on three-dimensional flow focusing using more than a single layer (Wolff et al. 2003; Sundararajan et al. 2004; Simonnet and Groisman 2005;
Yang et al. 2005; Chang et al. 2007; Tsai et al. 2008; Kummrow et al. 2009). Hairer and Vellekoop (2009), Park et al. (2009), and Mao et al. (2009) recently presented creative devices for flow focusing in a single layer using multiple sheathing inlets and inertial microfluidic effects.

Optical measurement techniques determining forward and side scattering, axial light absorbance and extinction alterations resulting from microbiological cell conditions or chemical sample concentrations are important methods for biotechnological lab-on-chips. One of the limiting factors for the detection sensitivity is the maximum light irradiance $I\left(\mathrm{~W} / \mathrm{m}^{2}\right)$ coupled into the optical detection channel region. Therefore, the geometric design and fabrication of the light guiding and focusing parts are critical for the performance of the system. In most instances found in the literature, on-chip cytometers comprise bare optical fibers (Malic and Kirk 2007; Bernini et al. 2006) or on-chip waveguides (Ruano-Lopez et al. 2006; Bliss et al. 2007) without additional lenses, where the light emerges coneshaped according to the numerical aperture (NA). To create a converging light path with a defined focal spot a broad range of solutions have been presented in recent years. They can be divided into two categories according to the optical light path orientation in relation to the substrate surface (in-plane or out-of-plane).

The combination of a light microscopic setup with a transparent chip material to measure out-of-plane luminescence is a widely distributed approach due to the simple handling and configurability. However, as one of the main criteria in microflow cytometry is size, there are multiple publications where out-of-plane microlenses are implemented, including mechanical actuation (Ren and $\mathrm{Wu}$ 2007), electromechanical actuation (Lee and Lee 2007), and electrowetting (Kuiper and Hendriks 2004). For in-plane measurements external elements can be added to achieve the focusing, like ball or cylindrical gradient index (GRIN) lenses or the focusing elements are integrated within the chip structure. Literature exists for the in-plane microlenses with different materials and arrangements, like polydimethylsiloxane (PDMS) (Camou et al. 2003) and SU-8 (Wang et al. 2004). However, if the presented lenses are in direct surface contact to the analysis channel the particle stream path might experience a distinctive deviation. As a first step toward a practical robust micro-optical flow cytometer a biconcave lens and polymer-based waveguides are integrated to increase the irradiance without interfering with the analytical channel flow profile. Recently, the proof-of-principle of a fluidic lens for in- and out-of-plane is successfully shown (Rosenauer and Vellekoop 2009).

In this publication the development, fabrication, and characterization of an optofluidic sensor device for microflow cytometry are presented (Fig. 1). The microfluidic device 


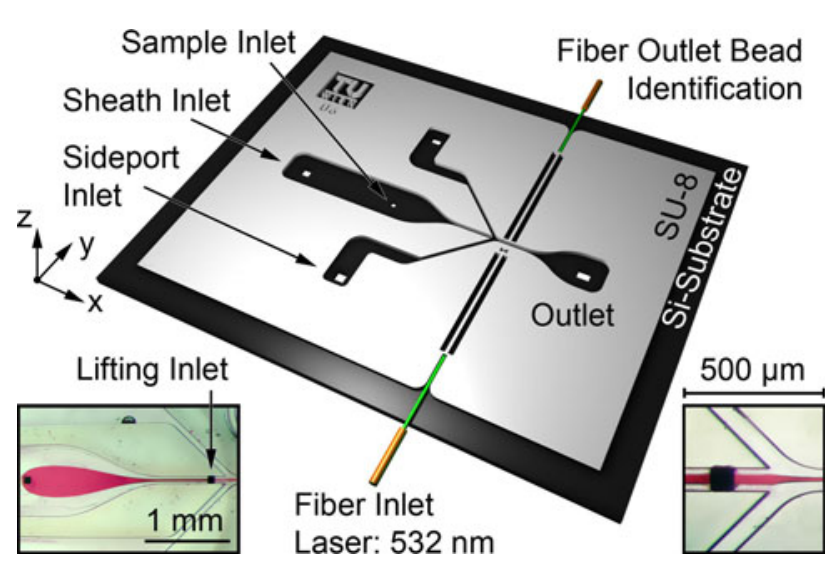

Fig. 1 Schematic of the absorbance-based integrated flow cytometer $\left(9 \times 13 \mathrm{~mm}^{2}\right)$ with micrographs of the hydrodynamic focusing region. Sample fluid is an aqueous colored ink solution; sheath fluid is deionized water

consists of a silicon substrate, a single-layer SU-8 channel structure, and a PDMS cover lid. The coaxial (horizontal/ vertical) sample alignment is achieved by reconfigurable three-dimensional hydrodynamic focusing in a single microstructured layer. Not only is it possible to achieve a wellfocused centered sample stream, the analyte can easily be steered independently to an arbitrary position in the channel. Even though this system provides high flexibility, it can be fabricated by standard lithographic methods without complex fabrication steps.

The measurement sensitivity is increased by the integration of a polymer lens in the optical path detached from the analysis channel geometry. In this contribution the detection setup comprises a standard solid-state laser and silicon photodiodes operated by lock-in-amplification as an alternative to single photon counting units. This research illustrates the applicability of compact low-cost/low-power elements for single cell/particle classification. The discrimination capabilities of the device are excellently displayed by the detection of three different-sized bead populations in a single suspension by analyzing signal drop height/width. The application of a custom-made plastics holder along with syringe pump control allows easy packaging and handling. After purchasing the photolithographic masks (two-mask process) both fabrication and packaging are simple and fast, allowing a whole device wafer to be fabricated and tested in 1 day.

\section{Experimental section}

\subsection{Materials}

For the fabrication of the device single-side polished 4-inch silicon wafers (lattice orientation ( 100$)$, thickness $360 \mu \mathrm{m}$ ) were purchased. The negative photoresist SU-8 2000 ( $n=1.60$ at $532 \mathrm{~nm}$, cross-linked SU-8) was supplied by Microchem (Newton, MA, USA), the PDMS by DowCorning (Corning, NY, USA), and both were used according to the manufacturer's instructions. A clear positive photoresist AZ6612 as sacrificial masking layer for the inlet etching steps and a resist developer AZ826 were purchased from AZ-Electronic Materials (Branchburg, NJ, USA). For wafer processing and cleansing, standard chemicals, including alkali hydroxide etchant- $\mathrm{KOH}$, propylene glycol monomethyl ether acetate-PGMEA, Ethanol, Isopropyl alcohol, Acetone, were used (Sigma-Aldrich, MO, USA). The microchip holder was fabricated in Polytetrafluorethylen PTFE (DuPont, Wilmington, USA) with press-fitted PTFE tubings (inner/outer diameter $=0.5 / 1.0 \mathrm{~mm}$ ) (Liquid-Scan, Ueberlingen, GER). For the purpose of comparison to biological cell assays three different sizes of transparent polystyrene calibration beads (Fluka particles, var. coeff. $=1.5 \%$, solid content $2 \%$, Sigma-Aldrich, MO, USA) were used in the experiments: $4( \pm 0.1) \mu \mathrm{m}, 8$ $( \pm 0.1) \mu \mathrm{m}$, and $12( \pm 0.2) \mu \mathrm{m}$, respectively. Rhodamine B (Roth, Karlsruhe, GER) was purchased for light path visualization to characterize the hydrodynamic and light focusing ability.

\subsection{Channel design and fabrication}

The two main innovative parts of the presented microflow cytometer are the easy-to-package single-layer 3D hydrodynamic focusing design and the conveniently placed integrated optical elements not influencing the microfluidic conditions in the channel (Fig. 1). In addition only miniaturized sensing elements instead of single photon counting units have been used for optical detection. In the optical inspection zone, the sample is surrounded and defined by a reconfigurable sheath flow. This is achieved in a series of independent sheathing steps producing a stable core flow even at low core flow rates $<0.1 \mu \mathrm{l} / \mathrm{min}$. Since all fluidic inlets are at the bottom of the microchannel, the device fabrication is a straight-forward photolithographic process. A schematic cross-section plot of the final device secured in the custom-made plastics holder is shown in Fig. 2a.

The optofluidic elements (channel, waveguide, and lens) were fabricated in negative photosensitive epoxy resist SU8 on a standard 4-inch silicon wafer. The latter was purchased with nitride coatings on both sides as protection and anti-oxidation layers. The fluidic inlet holes were anisotropically backside etched by $\mathrm{KOH}$ solution. To fabricate the mask structure for the etching process a thin film of positive photoresist AZ6612 (rotation speed 4,000 rpm for $15 \mathrm{~s}$, soft bake $105^{\circ} \mathrm{C}$ for $5 \mathrm{~min}$ ) was deposited and exposed using soda-lime photomasks (min. dimensions $>4 \mu \mathrm{m}$, tolerances $\pm 0.3 \mu \mathrm{m}$ ) purchased from Rose 


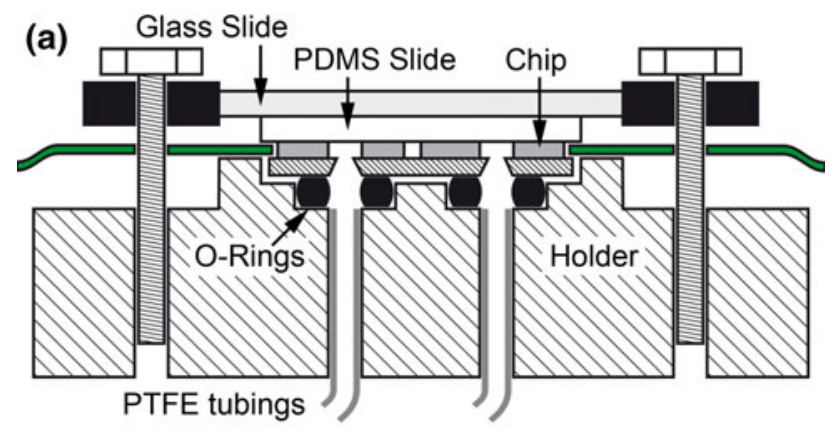

(b)

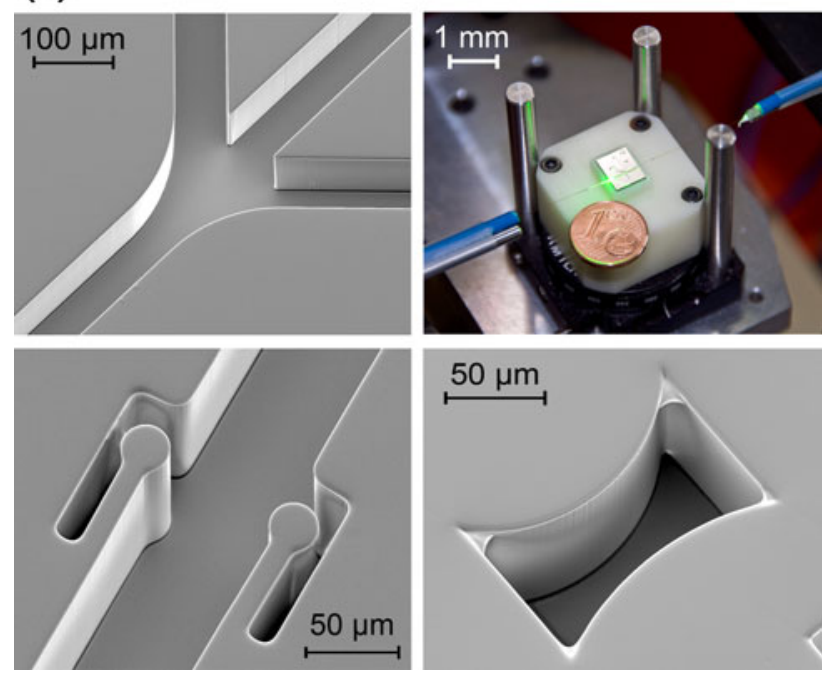

Fig. 2 a Schematics of fluidic and optical world-to-chip connection with plastics holder and clamped fiber/chip mounting. b Image of the microdevice with inserted glass fibers on top of the holder system incorporating the fluid inlets. The fourth mounting rod is removed for better visualization. Detailed SEM (scanning electron microscope) images of the hydrodynamic sideport region, the integrated fiber clamps, and the cylindrical air/resist lens realized in SU-8

Fotomasken (Bergisch-Gladbach, GER). The alignment was done with a standard mask-aligner Suss MicroTech MA150 (Garching, GER). Immediately after post-exposure bake $\left(60^{\circ} \mathrm{C}\right.$ for $\left.5 \mathrm{~min}\right)$ and development (AZ826 for $15 \mathrm{~s}$ ) the $\mathrm{KOH}$ etching steps were performed ( $7 \mathrm{~h}$ with $40 \% \mathrm{w} / \mathrm{w}$ $\mathrm{KOH}$ solution at $75^{\circ} \mathrm{C}$ ). The silicon nitride layer on the other side worked as an automatic etch stop.

SU-8 is a negative photoresist most sensitive to near ultra violet (near-UV) light. Due to low absorption high aspect ratios (thickness $>500 \mu \mathrm{m}$ with a single coating) and clean edges can be realized (Lorenz et al. 1998). For a better adhesion of the photoresist a sacrificial titanium layer with a thickness of $20 \AA$ was sputtered on the silicon wafer. For a homogeneous distribution the epoxy $(1 \mathrm{ml})$ was poured on the wafer at a spin rate of $500 \mathrm{rpm}$ for $20 \mathrm{~s}$. The immediately subsequent spin $(4,000 \mathrm{rpm}$ for $40 \mathrm{~s})$ generated a film thickness of $82 \mu \mathrm{m}$ measured with a DEKTAK 8 stylus profiler. After soft bake $\left(65^{\circ} \mathrm{C}\right.$ for $5 \mathrm{~min}$ and $95^{\circ} \mathrm{C}$ for $25 \mathrm{~min}$ ) and slow cooling (important to reduce material strains) edge bead removal was performed. Since the position and dimension of the lifting port inlet is crucial for the performance of the 3D particle focusing, the processed $\mathrm{Si}$ wafer comprising the inlet holes has to be perfectly backside-aligned for the SU-8 photolithographic step. Therefore, a vacuum contact between mask and wafer was obtained to prevent projection faults with the Double-Side Mask Aligner EVG620 (EVG, St. Florian am Inn, AUT). After cross-linking of the exposed resist (post-exposure bake for $12 \mathrm{~h}$ at $60^{\circ} \mathrm{C}$ ) the unexposed SU-8 was dissolved with PGMEA ( $8 \mathrm{~min}$ ). After the final step (hard bake at $150^{\circ} \mathrm{C}$ for $45 \mathrm{~min}$ ) the material is completely cross-linked achieving its chemical stability and mechanical strength. More details on the fabrication procedure can be found in Svasek et al. (2004). PDMS cover lids $\left(15 \times 15 \mathrm{~mm}^{2}\right.$, thickness $=0.5 \mathrm{~mm}$ ) were used as a waterproof gasket sheet on top of the microfluidic chip. The completed 4-inch wafer was diced resulting in 66 microfluidic devices $\left(9 \times 13 \mathrm{~mm}^{2}\right)$. Figure $2 \mathrm{~b}$ illustrates the results of the fabrication process. Besides the sideport junction and the integrated optical lens and waveguide, a close-up of the fiber-clamps is shown. To ensure a stable position of the inserted glass fibers during the whole measurement cycle, the heads of the clamping beam fix the fibers as the distance of the clamps is smaller than the width of the cladding. Since the application of optical adhesives is not necessary, chip and fibers can easily be reused.

\subsection{Fluidic and optical interconnection system}

As mentioned before the $\mathrm{Si} / \mathrm{SU}-8$ device was capped with a PDMS cover sheet bonded to a Pyrex glass slide. This transparent cover allowed external observation and visible detection of cells or particles under a microscope. To secure the setup against mechanical vibrations a new plastics chip holder system (Fig. 2a) was realized, which highly alleviates chip sealing and measurement repeatability. The glass fibers were mechanically fixed by the integrated fiber-clamps and the PDMS device sealing after insertion. The fiber trenches were tapered to ensure self-alignment and high coupling efficiency. The holder was fabricated in PTFE with pressfitted tubing and the interconnection between tubing and chip was achieved via rubber o-rings (inner/outer diameter $=0.8 / 1.8 \mathrm{~mm}$ ). Since the fiber-to-chip coupling needed to be mechanically secured and easily handled a manual probe measurement setup comprising a microscope and a micropositioning table was altered to obtain a reconfigurable fixation of the fibers mounted to the probe heads.

\subsection{Detection system}

A diode pumped solid-state laser $\left(\lambda=532 \mathrm{~nm}, P_{\mathrm{CW}}=\right.$ $20 \mathrm{~mW}, f_{\max }=30 \mathrm{kHz}$ ) was used to provide the incident 
light beam in the designed fiber-optic system. This freespace laser light was coupled in a glass fiber with reduced cladding (core/cladding $=50 / 70 \mu \mathrm{m} \pm 2 \mu \mathrm{m}, \mathrm{NA}=0.22$, Polymicro Technologies, AZ, USA) via a fixed aspheric lens collimator $(\lambda=543 \mathrm{~nm}, f=11.0 \mathrm{~mm}, \mathrm{NA}=0.25$, Thorlabs, NJ, USA). The cleaved fiber pigtails were inserted in the tapered fiber notches and fixed by the circumjacent clamps and the covering PDMS lid. The light intensity and the occurring axial light loss due to particle absorption and refraction was measured by a pre-amplified noise-suppressed Si-photodetector (350-1,100 nm, 0.65 A/W at $\lambda=970 \mathrm{~nm}, 70 \mathrm{~dB}$ ). The setup (laser and detector) was operated in lock-in-amplification (Perkin Elmer 7265 DualPhase $250 \mathrm{kHz}$, Signal Recovery, Wokingham, UK) at $30 \mathrm{kHz}$. The output of the lock-in-amplifier (extinction signal data) was recorded using the trigger events of the oscilloscope TDS2014 (Tektronix, OR, USA). The data acquisition and analysis software was written in MATLAB (The Mathworks, MA, USA).

\subsection{Assay protocol}

For the applied pressure-driven flow four syringe pumps (Chemyx Fusion 200, Chemyx, TX, USA) were used for one sample, one sheath, two sideports, and one lifting flow rate supply. The two sideport flow rates were adjusted with a dual syringe pump to achieve a stable and even flow rate symmetry. To avoid a priori introduction of air bubbles the chip and O-rings were first cleansed with ethanol. After aligning the microdevice onto the holder, the microchannels were filled with the sheath medium and sealed. Special attention was given to ensure that buffer and sheath media have matching densities to that of the particles, to minimize particle sedimentation effects in the tubing and syringes. (Note: In the microfluidic device a particle/fluid density mismatch, e.g., DI-water/polystyrene, does not affect the measurement data.) Since polystyrene particles ( $\rho=1.05 \mathrm{~g} / \mathrm{ml}, \mu=1.5 \mathrm{mPa} \mathrm{s}$ ) were applied to proof the performance of the design an aqueous solution of $14 \%$ (w/w) Sucrose (Sigma S9378-500g) in $0.2 \mu \mathrm{m}$ filtered deionized water was used. To achieve a similar particle count distribution of the three polystyrene bead sizes it was necessary to prepare the sample as follows: mixture of $1.55 \mu \mathrm{l}$ of the original $4 \mu \mathrm{m}$ bead suspension, $12.6 \mu \mathrm{l}$ of the $8 \mu \mathrm{m}$ suspension, $40 \mu \mathrm{l}$ of the $12 \mu \mathrm{m}$ suspension to achieve a ratio of 1:1:1 with approx. $8.8 \times 10^{5}$ particles per extraction volume. After 3-5 washing steps the beads were resuspended in $1.5 \mathrm{ml}$ density-matched buffer medium. The human cell line used for the viability experiment was Jurkat (an immortalized acute T-lymphocyte leukemia cell line) kindly provided by the Institute of Virology, Slovak Academy of Sciences (Bratislava, Slovakia). Jurkat cells were grown in culture flasks at $37^{\circ} \mathrm{C}$ and $5 \% \mathrm{CO}_{2}$.
DMEM containing $4.5 \mathrm{~g} / 1$ glucose, $2 \mathrm{mM}$ L-glutamine, $10 \%$ fetal calf serum and antibiotics (100 units/ml penicillin, $100 \mu \mathrm{g} / \mathrm{ml}$ streptomycin and $0.25 \mu \mathrm{g} / \mathrm{ml}$ amphotericin B) was used as medium, all purchased from Lonza Bioscience. Trypan blue (Roth, Karlsruhe, GER) was applied as vital stain.

\section{Results and discussion}

In this study, the fluidic and optical performance of the proposed microflow cytometer is investigated and its ability as on-chip solution experimentally demonstrated. In general, the main aspects in terms of applicability are simple chip packaging/handling, low-noise optical inspection, and stable particle alignment. Therefore, a reconfigurable hydrodynamic focusing approach is analyzed with a decreased influence of the inertial forces on the fluidic flow behavior (Carlo 2009). The particle inspection region features a novel on-chip lens increasing the irradiance. Since the biconcave lens is utilizing the refractive index difference between the transparent epoxy $(n=1.6)$ and air, the optical structure is detached from the microfluidic channel wall thus not altering the particle stream behavior. To create a suitable alternative system for fast bioanalytical tests, the off-chip detection setup comprises relatively simple low-cost elements (standard photodiode and laser source). This creates an integrated system which we believe to be very valuable for research labs and point-ofcare diagnostics (e.g., doctor's offices).

\subsection{D hydrodynamic sample focusing}

The fluidic condition of the pressure-driven flow system was numerically simulated using COMSOL Multiphysics (Comsol Inc., Palo Alto, USA) to fully understand the flow behavior and derive channel geometries to achieve ideal particle centering. Due to the pre-derived Reynolds number $R e \approx 1$ the complete Navier-Stokes equations were used for steady-state simulation of the incompressible isothermal fluid flow with convection and diffusion. All inlet flows were defined as laminar and fully developed (entrance length $=1 \mathrm{~m}$ ), and a no-slip condition at the channel walls was assumed. As parameter of a reliable simulation the diffusion coefficient was defined with $D=0.5 \times 10^{-9} \mathrm{~m}^{2} / \mathrm{s}$. The fluid's diffusive conductivity in our simulation was isotropic and, therefore, only denoted by the diffusion constant $D$.

The square inlet holes of the two sideports, the backside sheath flow, and the outlet have an edge length of $250 \mu \mathrm{m}$. For the inlet and lifting ports the silicon is etched through to an edge length of $80 \mu \mathrm{m}$. The channel width at the sample fluid entrance is $400 \mu \mathrm{m}$ and is narrowed down to $80 \mu \mathrm{m}$. 
The $45^{\circ}$ adjacent sideport channels are also $80 \mu \mathrm{m}$ broad. The lifting inlet covers the whole channel width to ensure a homogeneous lifting of the sample. The height of the SU-8 channel is $82 \mu \mathrm{m}$. The backside sheath fluid is introduced in a broad microchannel overflowing the sample inlet and constraining the sample to the bottom of the channel. A preliminary lateral focusing is achieved by narrowing the channel geometry to one-fifth of its original width (Fig. 3a). Due to three-sided sheathing at this point, clogging of the channel is inhibited. As a next step the sample stream is lifted to the center of the channel (Fig. 3b). Therefore, the position and dimensions of the lifting port inlet are crucial for correct particle centering. In a previously presented study of our group an undesired secondary effect of the lifting step has been discovered (Hairer et al. 2008). As the sample stream is lifted an expansion of the sample stream width due to the parabolic flow velocity profile is measured in correlation to the vertical position. Therefore, in the last step the sample is pinched off by the sideport streams to inhibit the broadening and ensure the centered position and the particle alignment. An additional practical feature of the coaxial bead alignment is the prevention of polystyrene particle contact on the hydrophobic surfaces of SU-8 and PDMS. With the applied flow rates $Q_{\text {Sample }}=1, Q_{\text {Sheath }}=50, Q_{\text {Sideports }}=5, Q_{\text {Lift }}=$ $20 \mu \mathrm{l} / \mathrm{min}$ a consistent cross-sectional spot of $12 \times 14 \mu \mathrm{m}^{2}$

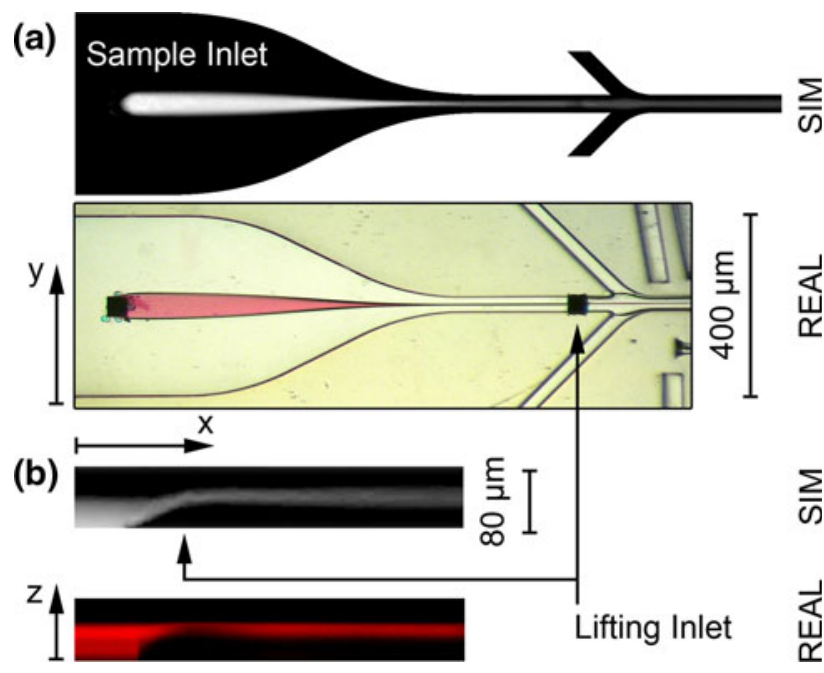

Fig. 3 Simulation results and light and confocal microscopy images of the hydrodynamic focusing of the microflow cytometer. a Horizontal sheathing of the sample stream. The intensity of the colored ink solution decreases after the lifting inlet due to the reduced height of the lifted sample stream, which is completely surrounded by the transparent sheath flow (DI-water) after the lifting. b Cross-sectional close-up of the lifting inlet and the following sideport junction illustrates the good agreement of theory and experiment of the sample centering. The difference between simulation and practical approach suggests that the lifting procedure is more abrupt than predicted. A possible reason could be the slightly rough edges of the inlet holes of the $\mathrm{SiN}$ and the substrate (width $\times$ height) is achieved both in computational fluid dynamics simulation and experiments. Detailed results are depicted in Fig. 3. The horizontal (XY) micrograph shows the hydrodynamic focusing using a highly concentrated aqueous ink solution. The cross-sectional (XZ) image is recorded using a confocal microscope (Nikon Eclipse C1 Plus, Nikon, Japan) with a $20 \times$ objective (Plan Apo Dry $20 \times / 0.75$ WD 1.0 , Nikon, Japan). The image resolution was $2,048 \times 2,048$ pixels with a Z-step size of $0.44 \mu \mathrm{m}$ and a pixel dwell time of $3.12 \mu \mathrm{s}$. A green $\mathrm{HeNe}$ laser $(P=2 \mathrm{~mW}, \lambda=543 \mathrm{~nm})$ in combination with a spectral long pass filter (570 LP) was used. $50 \mu \mathrm{M}$ of Rhodamine B was dissolved in $94 \%(\mathrm{w} / \mathrm{w})$ ethanol to create a fluorescent sample fluid. In general, the use of ethanol is helpful to inhibit/prevent the creation of air inclusion in the channels and to increase the luminescent quantum yield and lifetime (Snare et al. 1982). A comparison of the two cross-sections shows very good agreement of the captured confocal image and the simulation result. In conclusion, the achieved sample stream dimensions in combination with the parabolic flow velocity profile in the inspection zone $(v=0.21 \mathrm{~m} / \mathrm{s})$ are sufficient enough to align the bead suspension. A more detailed description of vertical and horizontal sample shifting with a similar reconfigurable device developed by our group can be found in Hairer and Vellekoop (2009).

\subsection{Optical inspection with integrated polymer-based elements}

In the center part of the chip several optical elements are integrated in the transparent polymer. After alignment the hydrodynamically centered particles are driven through this inspection region. As a first step the excitation light is coupled via the fixed aspheric lens fiber coupler from the free-space laser into the multimode glass fiber. The laser head and the FC/PC collimator are mounted and adjusted in a 1-inch cage system from Thorlabs (Thorlabs Inc., NJ, USA). The laser-fiber coupling loss yielded $1.18 \mathrm{~dB}$ determined by a laser power meter (Ophir Nova II, USA). After insertion and fixation of the cleaved fiber ending in the microchip the light is coupled into the on-chip polymer waveguides and guided by total internal reflection. The refractive index of SU-8 which forms the waveguide core is $n=1.60$, and the surrounding materials (lateral: air $n=1$, bottom: $\mathrm{SiO}_{2} n=1.46$ and a reflective titanium layer, top: PDMS $n=1.43$ ) enable waveguiding. The light exiting the waveguide is focused by a biconcave air/resist lens and coupled into the microfluidic channel (Fig. 4). This subsequent cylindrical lens $\left(d=\right.$ thickness, $R_{1}$ and $R_{2}=$ left and right curvature radii) is designed using simple lens maker's equations combined with the imaging properties 
$\frac{1}{f}=\frac{n_{2}-n_{1}}{n_{1}}\left(\frac{1}{R_{1}}+\frac{1}{R_{2}}\right)+\left(\frac{\left(n_{2}-n_{1}\right)^{2} d}{n_{2} R_{1} R_{2}}\right)$

with $n_{2}=1.6, n_{1}=1, R_{1}=160 \mu \mathrm{m}, R_{2}=100 \mu \mathrm{m}$ and $d=50 \mu \mathrm{m}$ (Fig. 4b). A theoretical focal length of $95.6 \mu \mathrm{m}$ is achieved.

To proof and simulate the calculations and emerging variations due to fabrication issues raytracing experiments with the dedicated software ZEMAX EE (ZEMAX, WA, USA) were conducted to focus the incident light beam to the center of the microfluidic channel (Fig. 4a). Since the waveguide-exiting light rays are divergent, the first cylindrical lens curvature $\left(R_{1}\right)$ is primarily used to collimate the light whereas the second plane $\left(R_{2}\right)$ is applied to generate the converging light path. To our knowledge, this is the first axial SU-8 lens not influencing the analytic channel integrated in a microflow cytometer. As a result the channel geometries can be further downsized enabling a more precise hydrodynamic focusing. The integration of a cylindrical 2D lens in the channel wall at these dimensions $80 \times 80 \mu \mathrm{m}^{2}$ would result in strong disturbances of the flow stream conditions and, therefore, a decrease in signal quality. The excitation light path was visualized in two ways. First, since SU-8 has a strong autofluorescence when excited with the applied laser wavelength $(\lambda=532 \mathrm{~nm})$ (Ong et al. 2007) the incident laser light traveling through the waveguide and the biconcave lens is visualized. Second, the microfluidic channel is filled with a low-concentrated fluorochrome (Rhodamine B, excitation $532 \mathrm{~nm}$, emission at $600 \pm 20 \mathrm{~nm}$ ) to display the focal plane inside the channel. The background was illuminated with a conventional cold light source to visualize the channel walls
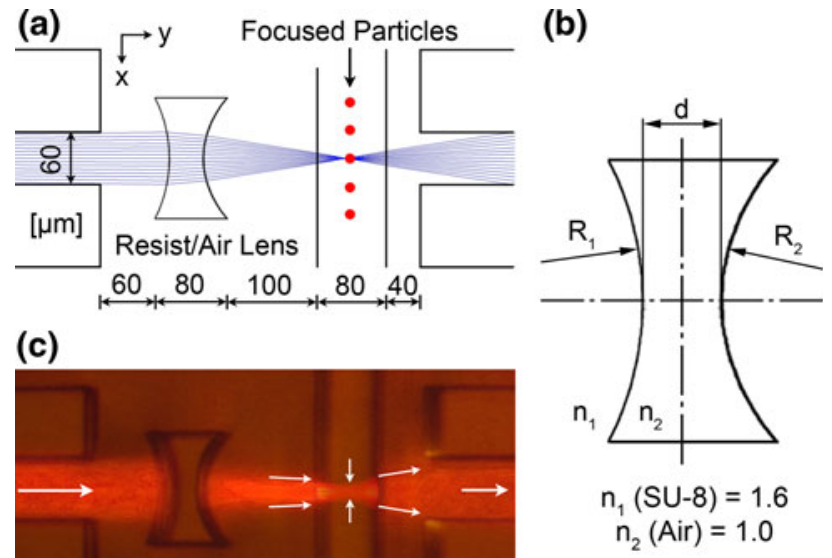

(c)

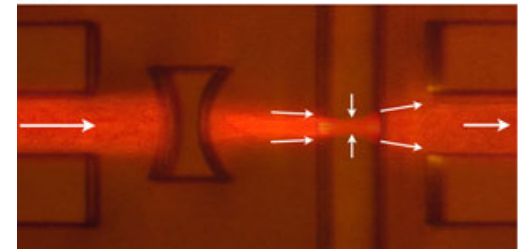

$\mathrm{n}_{2}($ Air $)=1.0$

Fig. 4 a Raytracing simulation of the light focusing. b Biconcave lens geometry with two different curvature radii. c Superimposed micrograph of the optical inspection region with integrated waveguides and 2D lens. The auto-fluorescence of SU-8 in combination with the Rhodamine B solution in the sample channel is utilized to visualize the light path and the integrated lens. The fluorescent micrographs are superimposed and illustrate the excellent agreement of the raytracing simulation and experiments (Fig. 4c). The created lens plane width (XY) in the center of the channel is $15 \mu \mathrm{m}$ which means that an almost optimal spotsize for particle measurements $(4,8,12 \mu \mathrm{m})$ is achieved. In a quantitative comparison with a microdevice without the lens/waveguide structure an improvement in coupling efficiency by $13 \mathrm{~dB}$ was measured. Despite the focused laser beam and increased irradiance, no heating effects in the channel/material was observed. In a recently published proof-of-principle study (Buchegger et al. 2009) we implemented a 2D fluidic lens based on the same principle but with the ability to shift the focal point in the channel according to the used fluid. This method is very promising to overcome fabricational variations. Furthermore, proofof-principle measurements of single fluorophore-coated beads were conducted, and a satisfactory performance in single particle detection without the use of single photon counting units was achieved. Work is in progress to further increase the coupling efficiency and minimize the focal spot size in three dimensions. A promising method for incorporating a lens with the ability to focus the incident light three-dimensionally is the implementation of an optofluidic lens (Rosenauer and Vellekoop 2009).

\subsection{Identifications of polystyrene microspheres}

The optical investigation of bead samples comprises multiple different observation techniques depending on the preparation of the sample and the evaluated parameters, such as fluorescence, forward/side scattering (FSC/SSC), and extinction. In this study the signal extinction as a parameter for bead size and transparency was analyzed. FSC devices for small and large angle scattering were also realized on the silicon wafer. $5-10^{\circ}$ inclined on-chip waveguides were integrated to enable FSC measurements and increase the biochemical parameter measurement range and the sensitivity by signal correlation of two FSC waveguides.

The waveguide-fiber coupling arrangement from the excitation path was also placed on the opposite side of the channel as detector path, with the absorbance signal traveling through the analyte channel and a second on-chip waveguide, and being decoupled into a detection glass fiber. The light intensity was then measured by a standard Si-photodetector (30 dB pre-amplified) operated in lock-inamplification $(f=30 \mathrm{kHz})$. By applying standard low-cost optical elements capable of being integrated this setup differs from other approaches which use single photon counting units to detect bead parameters with a possible miniaturizing trade-off. In order to evaluate the performance of the microflow cytometer different particle sizes 
were centered and analyzed. Polystyrene particle samples $(d=4 / 8 / 12 \mu \mathrm{m})$ were used with the given concentrations $\left(c=5.7 \times 10^{8} / 7.0 \times 10^{7} / 2.2 \times 10^{7}\right.$ beads $\left./ \mathrm{ml}\right)$ and the pre-defined flow rates (Sheath/Sample/Lifting/Sideport $=5 / 0.1 / 2 / 0.5 \mu 1 / \mathrm{min}$ ). In the microdevice it is essential that sample cells/particles are measured individually. In relation to our achieved hydrodynamic sample spot size of $12 \times 14 \mu \mathrm{m}^{2}$ the maximum concentration applied should not exceed $c=3 \times 10^{8}$ beads $/ \mathrm{ml}$ to reach a theoretical distance of $20 \mu \mathrm{m}$ between the particles. The microbeads were suspended in the mentioned density-matched solution (see Sect. 2.1). Afterward, the suspension was driven through the microfluidic chip by applying the pre-simulated flow rates. Consequently, as a centered single bead passes through the light beam, a detectable amount of light is blocked depending on its size. The lock-in-amplifier output was displayed on the oscilloscope in AC-coupling mode, minimizing low-frequency signal drift $(<5 \mathrm{~Hz})$ and showing a distinct negative peak for each particle at a single trigger event. For different-sized particles multiple negative peak voltages were obtained excellently assigning the bead type (size) to the voltage peak height. In order to test the chip functionality a low-concentrated suspension with 4 and $12 \mu \mathrm{m}$ polystyrene particle was mixed. Figure 5a shows the extinction measurement results (negative edge trigger level $=-0.015 \mathrm{~V}$ ). A distinct difference in peak height (average voltage $4 \mu \mathrm{m}=-0.04 \mathrm{~V}$; $12 \mu \mathrm{m}=-0.14 \mathrm{~V}$ was detected, allowing simple differentiation between two particle sizes. A close-up of a single signal drop is displayed in Fig. 1 ESM. A minimum detectable particle size of $\geq 4 \mu \mathrm{m}$ was achieved. In order to extend the measurement range and sensitivity the integration of novel optofluidic elements is planned.

To determine the discrimination capability of the presented microflow cytometer a $1.5 \mathrm{ml}$ suspension of 4,8 , and $12 \mu \mathrm{m}$ particles was mixed at bead ratio of 1:1:1 with approx. $8.8 \times 10^{5}$ beads per lot. A micrograph of the bead suspension is shown in Fig. 2 ESM. After extinction peak

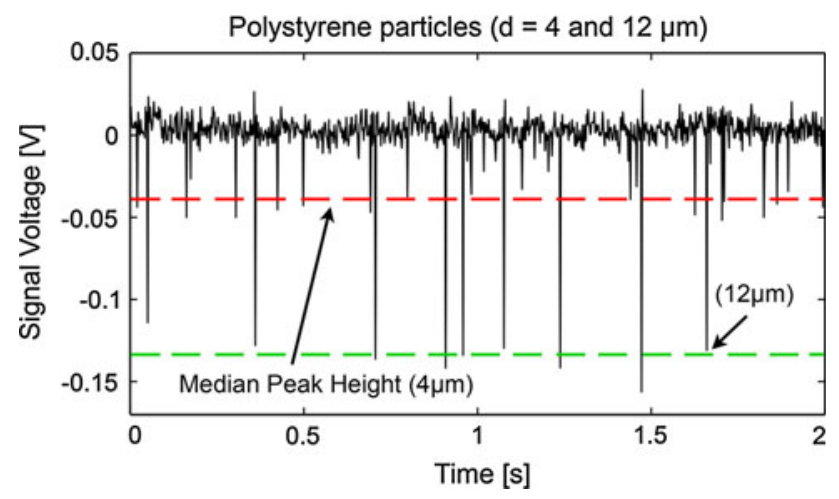

Fig. 5 Dynamic axial light loss measurements with two distinct extinction peak heights generated by different-sized polystyrene particles $(d=4$ and $12 \mu \mathrm{m})$ data collection of around 1,000 trigger events per single test run, a data processing program based on MATLAB was developed to automatically determine the peak height and width of every single event for a statistical analysis. In contrast to other approaches the bead suspensions were not measured separately, and therefore, the determined signal intensities from the measured microspheres were plotted in a single histogram (Fig. 6a). The distributions of the particle sizes show clear separation in three main areas, and Gaussian curve fits were calculated and added to the graph. From these distributions, the mean values $\mu$, the standard deviations $\sigma$, and the coefficients of variations (CV), defined as the standard deviation divided by the mean values of the signal peaks, were determined and listed in Table 1. To evaluate the influence of doublets the populations were investigated individually (Fig. 3 ESM). Each population showed 1-3\% occurence of cojoined polystyrene particles.

Even though the CV of the microflow cytometer is relatively high in comparison to certain commercial benchtop cytometers (Shapiro 2003), it is comparable to other integrated versions (Wang et al. 2004; Chen and Wang 2009). The reasons for the elevated CV for $4 \mu \mathrm{m}$ beads might be found in the slightly pulsed pumping motion of the syringe pumps and the more variably excited particles as a result of the slightly deviating center position of the $4 \mu \mathrm{m}$ beads. However, the presented low-cost setup enables a reliable particle differentiation only by peak height detection. From a total data count of 1,000 events, 278 peaks were classified as $4 \mu \mathrm{m}$ beads, 369 peaks as $8 \mu \mathrm{m}$, and 353 peaks as $12 \mu \mathrm{m}$, respectively. The reason for the deviating $4 \mu \mathrm{m}$ population might be due to observational error in the process of manually suspending the microbeads, which naturally leads to an increased deviation for the smaller particles suspensions with a higher bead concentration.

For a more detailed analysis the peak width of every event was also determined and plotted against the peak height (Fig. 6b).

Each population represents the microsphere's size as it is directly related to the axial light loss thus increasing the peak height and width.

With the investigation of the $4 \mu \mathrm{m}$ particle suspension the limit for this measurement setup and chip design is defined. As mentioned before, by integrating $5-10^{\circ}$ inclined on-chip waveguides FSC measurements would be enabled, and an increase of the biochemical parameter measurement range and the sensitivity by signal correlation of two FSC waveguides is expected.

\subsection{Viability bioassay}

In the final section the applicability of the microsystem for clinical assays is validated. Therefore, a comparable 


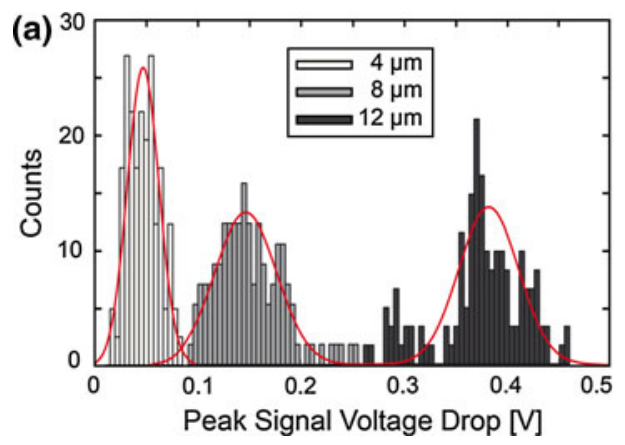

Fig. 6 a Statistical evaluation histograms of the axial light loss measurement data. Counts/signal-voltage measurement of $4 / 8 / 12 \mu \mathrm{m}$ (1:1:1) polystyrene particles in a single suspension. The diameter is directly correlated to the signal voltage peak height. The overlapping signal and additional peaks in the transition region between to distributions are a result of particle clustering (doublets and triplets) as they pass simultaneously through the detection region.

Table 1 Probability distribution parameters ( $\mu$ mean value and $\sigma$ standard deviation) for the different polystyrene particle samples

\begin{tabular}{lllc}
\hline Size $(\mu \mathrm{m})$ & $\mu(\mathrm{V})$ & $\sigma(\mathrm{V})$ & $\mathrm{CV}(\%)$ \\
\hline 4 & -0.047 & 0.0154 & 32.9 \\
8 & -0.139 & 0.0265 & 19.1 \\
12 & -0.375 & 0.0317 & 8.4 \\
\hline
\end{tabular}

standard viability test of T-lymphocyte cells (Jurkat cell line) was conducted by trypan blue staining. Since trypan blue is a vital dye, it does not interact with single cells unless the membrane is damaged. A dead or dying cell with a porous membrane is consequently stained blue and exhibits a higher absorption for the extinction measurements. Usually these clinical tests result in a viability count of 0.9 or higher. To obtain more significant extinction data allowing the characterization of the setup, a suspension with an increased amount of dead cells was prepared. After cultivation and suspension of the cells (see Sect. 2.5) a $0.4 \%$ trypan blue solution was used to selectively color the dead cells blue. To minimize optical interferences at the fluid interfaces (sample/sheath flow) the buffer medium was also used as surrounding fluid. The cell suspension was introduced to chip under the same fluid and optical condition as the particles mentioned before. The microflow cytometric data are again displayed in a frequency histogram providing two distinguishable distributions of viable and dead cells (Fig. 7). To validate the performance of the cytometer, the viability was also measured with a hemocytometer (0.71). The measurement of the microflow cytometer (0.69) was in good agreement with the conducted standard reference viability measurement. Work is in progress to further investigate the ability of the microsystem to be applied for clinical assays.

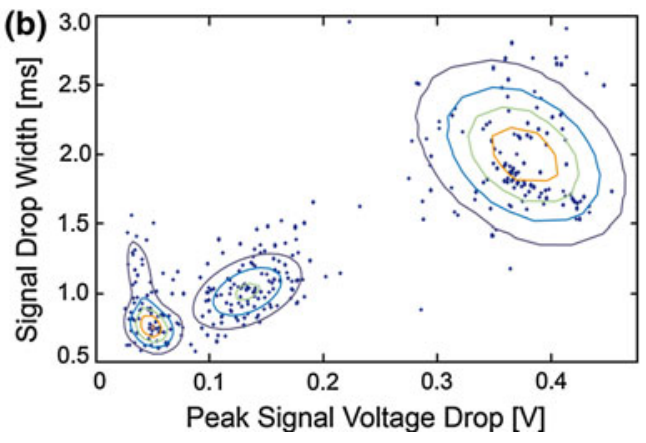

b Microsphere identification plots (2D) for three different-sized sets. Each point represents a single event with the determined pulse height and width. By constructing Gaussian mixture distributions of the signal data with three distinct center points the particle sets were successfully clustered. Although, a non-linear behavior between size and signal values (peak height and width) was determined, the discrimination was accomplished with an $89 \%$ accuracy

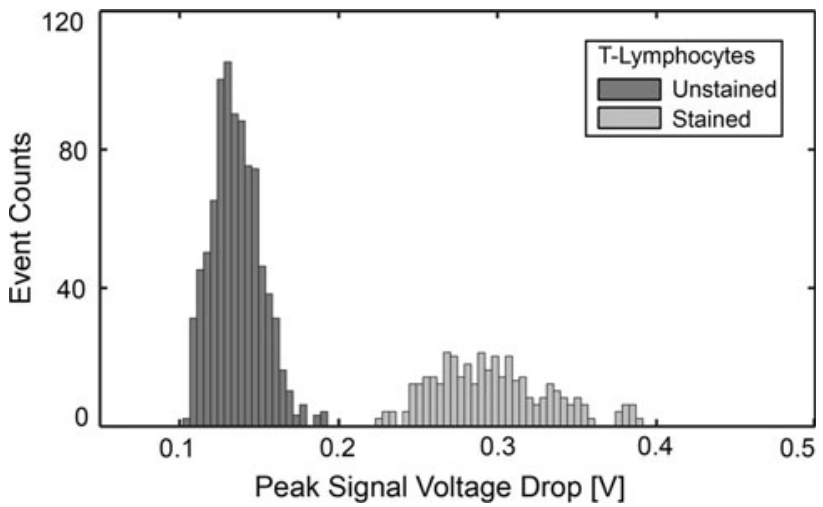

Fig. 7 Frequency histogram of a viability test of T-lymphocytes (Jurkat cells) by measuring the axial light loss combined with trypan blue staining. When setting the discrimination threshold to $V=0.2 \mathrm{~V}$, a viability of 0.69 is obtained

\section{Conclusion}

A single-layer microflow cytometer based on 3D hydrodynamic focusing with integrated polymer optical elements demonstrated the capability to distinguish different sets of monodisperse polystyrene beads. The microfluidic system and all the optofluidic elements were fabricated in a singlelayer photopolymer (SU-8) by standard lithography. Since the device was not capped permanently an entire wafer with over 60 devices could be completed in a single day. Each device could also be disassembled and thoroughly cleaned after every assay. The detection setup comprising off-chip standard devices like laserdiodes, multimode glass fibers, and a Si-photodetector was designed to contain lowcost equipment to facilitate simple reconfigurations for various types of assays and possible integration of those elements in future developments. Three different-sized polystyrene beads were tested by measuring the axial light loss of the incident light beam. The signal drop was 
evaluated by determining the peak height and width. The acquired data were statistically analyzed and allowed an excellent discrimination between the three populations. To display the bio-applicability of the presented microflow cytometer a standard viability test with T-lymphocyte cells was conducted. The frequency histogram shows two distinct distributions illustrating the great potential of this microfluidic cytometer in clinical assay automation.

These studies demonstrate the proof-of-principle capability for performing cytometric measurements on a lowcost level without compromising on the demanded sensitivity and reliability. This holds great promise for further development in terms of multiple parameter assays, cell analysis, and automated point-of-care diagnostics.

Acknowledgments For the sensor fabrication and the technical support we thank E. Svasek and P. Svasek (ISAS Technology Lab and ZMNS, Center of Micro- and Nanostructures, Vienna University of Technology). The SEM image analysis was carried out using facilities at the University Service Centre for Transmission Electron Microscopy (USTEM, Vienna University of Technology). This study was supported by grants from the Hochschuljubilaeumsstiftung der Stadt Wien 2008, and by project grant DSF.7135 of the Dutch STW Foundation to P. Verhaert. This project is also a part of the EU Marie Curie Research Training Network (MRTN) 'On-Chip Cell Handling and Analysis' CellCheck, project no. MRTN-CT-2006-035854.

Open Access This article is distributed under the terms of the Creative Commons Attribution Noncommercial License which permits any noncommercial use, distribution, and reproduction in any medium, provided the original author(s) and source are credited.

\section{References}

Ateya DA, Erickson JS, Howell PB, Hilliard LR, Golden JP, Ligler FS (2008) The good, the bad, and the tiny: a review of microflow cytometry. Anal Bioanal Chem 391(5):1485-1498

Bernini R, Nuccio ED, Brescia F, Minardo A, Zeni L, Sarro PM, Palumbo R, Scarfi MR (2006) Development and characterization of an integrated silicon micro flow cytometer. Anal Bioanal Chem 386(5):1267-1272

Blankenstein G, Darling Larsen U (1998) Modular concept of a laboratory on a chip for chemical and biochemical analysis. Biosens Bioelectron 13(3-4):427-438

Bliss CL, McMullin JN, Backhouse CJ (2007) Rapid fabrication of a microfluidic device with integrated optical waveguides for DNA fragment analysis. Lab Chip 7(10):1280-1287

Blow N (2007) Microfluidics: in search of a killer application. Nat Meth 4(8):665-670

Buchegger W, Rosenauer M, Vellekoop MJ (2009) Microfluidic measurement system for fluorescent particles with three-dimensional sheath flow and a self-aligned adjustable microlens. Procedia Chemistry 1(1):1123-1126

Camou S, Fujita H, Fujii T (2003) Pdms 2d optical lens integrated with microfluidic channels: principle and characterization. Lab Chip 3(1):40-45

Carlo DD (2009) Inertial microfluidics. Lab Chip 9(21):3038-3046

Chang CC, Huang ZX, Yang RJ (2007) Three-dimensional hydrodynamic focusing in two-layer polydimethylsiloxane (PDMS) microchannels. J Micromech Microeng 17(8):1479-1486
Chen HT, Wang YN (2009) Optical microflow cytometer for particle counting, sizing and fluorescence detection. Microfluid Nanofluid 6(4):529-537

Dittrich PS, Tachikawa K, Manz A (2006) Micro total analysis systems. Latest advancements and trends. Anal Chem 78(12): 3887-3908

Fu LM, Yang RJ, Lin CH, Pan YJ, Lee GB (2004) Electrokinetically driven micro flowcytometers with integrated fiber optics for online cell/particle detection. Anal ChimActa 507(1):163-169

Fu LM, Tsai CH, Lin CH (2008) A high-discernment microflow cytometer with microweir structure. Electrophoresis 29(9): 1874-1880

Golden JP, Kim JS, Erickson JS, Hilliard LR, Howell PB, Anderson GP, Nasir M, Ligler FS (2009) Multi-wavelength microflow cytometer using groove-generated sheath flow. Lab Chip 9(13):1942-1950

Hairer G, Vellekoop M (2009) An integrated flow-cell for full sample stream control. Microfluid Nanofluid 7(5):647-658

Hairer G, Paerr G, Svasek P, Jachimowicz A, Vellekoop M (2008) Investigations of micrometer sample stream profiles in a threedimensional hydrodynamic focusing device. Sens Actuators B Chem 132(2):518-524

Huh D, Gu W, Kamotani Y, Grotberg JB, Takayama S (2005) Microfluidics for flow cytometric analysis of cells and particles. Physiol Meas 26(3):R73-R98

Janasek D, Franzke J, Manz A (2006) Scaling and the design of miniaturized chemical-analysis systems. Nature 442(7101): 374-380

Kuiper S, Hendriks BHW (2004) Variable-focus liquid lens for miniature cameras. Appl Phys Lett 85(7):1128-1130

Kummrow A, Theisen J, Frankowski M, Tuchscheerer A, Yildirim H, Brattke K, Schmidt M, Neukammer J (2009) Microfluidic structures for flow cytometric analysis of hydrodynamically focussed blood cells fabricated by ultraprecision micromachining. Lab Chip 9(7):972-981

Lee SW, Lee SS (2007) Focal tunable liquid lens integrated with an electromagnetic actuator. Appl Phys Lett 90(12):121, 129-133

Lorenz H, Despont M, Fahrni N, Brugger J, Vettiger P, Renaud P (1998) High-aspect-ratio, ultrathick, negative-tone near-uv photoresist and its applications for mems. Sens Actuators A Phys 64(1):33-39

Malic L, Kirk AG (2007) Integrated miniaturized optical detection platform for fluorescence and absorption spectroscopy. Sens Actuators A Phys 135(2):515-524

Mao X, Lin SCS, Dong C, Huang TJ (2009) Single-layer planar on-chip flow cytometer using microfluidic drifting based three-dimensional (3d) hydrodynamic focusing. Lab Chip 9(11):1583-1589

McClain MA, Culbertson CT, Jacobson SC, Ramsey JM (2001) Flow cytometry of escherichia coli on microfluidic devices. Anal Chem 73(21):5334-5338

McDonald JC, Duffy DC, Anderson JR, Chiu DT, Wu H, Schueller OJA, Whitesides GM (2000) Fabrication of microfluidic systems in poly(dimethylsiloxane). Electrophoresis 21(1):27-40

Mogensen KB, Klank H, Kutter JP (2004) Recent developments in detection for microfluidic systems. Electrophoresis 25(21-22): 3498-3512

Myers FB, Lee LP (2008) Innovations in optical microfluidic technologies for point-of-care diagnostics. Lab Chip 8(12):2015-2031

Ong BH, Yuan X, Tan YY, Irawan R, Fang X, Zhang L, Tjin SC (2007) Two-layered metallic film-induced surface plasmon polariton for fluorescence emission enhancement in on-chip waveguide. Lab Chip 7(4):506-512

Park JS, Song SH, Jung HI (2009) Continuous focusing of microparticles using inertial lift force and vorticity via multi-orifice microfluidic channels. Lab Chip 9(7):939-948 
Ren H, Wu ST (2007) Variable-focus liquid lens. Opt Express 15(10):5931-5936

Ros A, Hellmich W, Regtmeier J, Duong TT, Anselmetti D (2006) Bioanalysis in structured microfluidic systems. Electrophoresis 27(13):2651-2658

Rosenauer M, Vellekoop MJ (2009) 3D fluidic lens shaping-a multiconvex hydrodynamically adjustable optofluidic microlens. Lab Chip 9(8):1040-1042

Ruano-Lopez J, Aguirregabiria M, Tijero M, Arroyo M, Elizalde J, Berganzo J, Aranburu I, Blanco F, Mayora K (2006) A new su-8 process to integrate buried waveguides and sealed microchannels for a lab-on-a-chip. Sens Actuators B Chem 114(1):542-551

Shapiro HM (2003) Practical Flow Cytometry, 4th edn. Wiley \& Sons

Simonnet C, Groisman A (2005) Two-dimensional hydrodynamic focusing in a simple microfluidic device. Appl Phys Lett 87(11):114104-1-114104-3

Sims CE, Allbritton NL (2007) Analysis of single mammalian cells on-chip. Lab Chip 7(4):423-440

Snare MJ, Treloar FE, Ghiggino KP, Thistlethwaite PJ (1982) Photophysics of rhodamine b. J Photochem 18(4):335-346

Sundararajan N, Pio MS, Lee LP, Berlin AA (2004) Three-dimensional hydrodynamic focusing in polydimethylsiloxane (PDMS) microchannels. J Microelectromech Syst 13(4):559-567

Svasek P, Svasek E, Lendl B, Vellekoop M (2004) Fabrication of miniaturized fluidic devices using su- 8 based lithography and low temperature wafer bonding. Sens Actuators A Phys 115(23):591-599

Tsai CH, Hou HH, Fu LM (2008) An optimal three-dimensional focusing technique for micro-flow cytometers. Microfluid Nanofluid 5(6):827-836

Tung YC, Zhang M, Lin CT, Kurabayashi K, Skerlos SJ (2004) Pdms-based opto-fluidic micro flow cytometer with two-color, multi-angle fluorescence detection capability using pin photodiodes. Sens Actuators B Chem 98(2-3):356-367

Wang CH, Lee GB (2006) Pneumatically driven peristaltic micropumps utilizing serpentine-shape channels. J Micromech Microeng 16(2):341-348

Wang Z, El-Ali J, Engelund M, Gotsaed T, Perch-Nielsen IR, Mogensen KB, Snakenborg D, Kutter JP, Wolff A (2004) Measurements of scattered light on a microchip flow cytometer with integrated polymer based optical elements. Lab Chip 4(4):372-377

Whitesides GM (2006) The origins and the future of microfluidics. Nature 442(7101):368-373

Wolff A, Perch-Nielsen IR, Larsen UD, Friis P, Goranovic G, Poulsen CR, Kutter JP, Telleman P (2003) Integrating advanced functionality in a microfabricated high-throughput fluorescent-activated cell sorter. Lab Chip 3(1):22-27

Wu X, Chon CH, Wang YN, Kang Y, Li D (2008) Simultaneous particle counting and detecting on a chip. Lab Chip 8(11):1943-1949

Xuan X, Li D (2005) Focused electrophoretic motion and selected electrokinetic dispensing of particles and cells in cross-microchannels. Electrophoresis 26(18):3552-3560

Yang R, Feeback DL, Wang W (2005) Microfabrication and test of a three-dimensional polymer hydro-focusing unit for flow cytometry applications. Sens Actuators A Phys 118(2):259-267

Yu C, Vykoukal J, Vykoukal DM, Schwartz JA, Shi L, Gascoyne PRC (2005) A three-dimensional dielectrophoretic particle focusing channel for microcytometry applications. J Microelectromech Syst 14(3):480-487

Zhang H, Chon C, Pan X, Li D (2009) Methods for counting particles in microfluidic applications. Microfluid Nanofluid 7(6):739-749 